\title{
On an open problem of Green and Losonczy: exact enumeration of freely braided permutations
}

\author{
Toufik Mansour \\ Department of Mathematics, University of Haifa, 31905 Haifa, Israel \\ toufik@math.haifa.ac.il
}

received Nov 25, 2003, revised Feb 2, 2004, Oct 25, 2004, accepted Oct 25, 2004.

Recently, Green and Losonczy [5, 6] introduced freely braided permutations as a special class of restricted permutations that has arisen in the study of Schubert varieties. They suggest as an open problem to enumerate the number of freely braided permutations in $S_{n}$. In this paper, we prove that the generating function for the number of freely braided permutations in $S_{n}$ is given by

$$
\frac{1-3 x-2 x^{2}+(1+x) \sqrt{1-4 x}}{1-4 x-x^{2}+\left(1-x^{2}\right) \sqrt{1-4 x}}
$$

Keywords: restricted permutations, freely braided permutations, generating functions

\section{Introduction}

Let $\alpha \in S_{n}$ and $\tau \in S_{k}$ be two permutations. Then $\alpha$ contains $\tau$ if there exists a subsequence $1 \leq i_{1}<$ $i_{2}<\cdots<i_{k} \leq n$ such that $\left(\alpha_{i_{1}}, \ldots, \alpha_{i_{k}}\right)$ is order-isomorphic to $\tau$; in such a context $\tau$ is usually called a pattern; $\alpha$ avoids $\tau$, or is $\tau$-avoiding, if $\alpha$ does not contain such a subsequence. The set of all $\tau$-avoiding permutations in $S_{n}$ is denoted by $S_{n}(\tau)$. For a collection of patterns $T, \alpha$ avoids $T$ if $\alpha$ avoids all $\tau \in T$; the corresponding subset of $S_{n}$ is denoted by $S_{n}(T)$.

One important and often difficult problem in the study of restricted permutations is the enumeration problem: given a set $T$ of permutations, enumerate the set $S_{n}(T)$ consisting of those permutations in $S_{n}$ which avoid every element of $T$. The first systematic study was not undertaken until 1985, when Simion and Schmidt [15] solved the enumeration problem for every $T \subseteq S_{3}$. More recent work on various instances of the enumeration problem may be found in [1], [2], [3], [4], [7], [8], [9], [10], [11], [12], [13], [14] and the references therein, [16], [17], [18], [19], and [20].

Recently, a special class of restricted permutations has arisen in the study of Schubert varieties. Green and Losonczy [5] defined, for any simply laced Coxeter group, a subset of "freely braided elements" (for details, see [5] and [6]), and they suggest as an open problem to enumerate the number of freely-braided permutations in $S_{n}$. 
Definition 1.1. A permutation $\pi$ is said to be freely-braided if and only if $\pi$ avoids each of the four patterns $1234,1243,1324$, and 2134. We denote the set of all freely-braided permutations in $S_{n}$ by $\mathcal{F}_{n}$, i.e., $\mathcal{F}_{n}=S_{n}(1234,1243,1324,2134)$.

Remark 1.2. In [5], a permutation $\pi$ is "freely braided" if and only if $\pi$ avoids each of the four patterns 4321, 3421, 4231, and 4312. Note, however, that a permutation $\pi$ avoids these four patterns if and only if $r(\pi)$ avoids each of the four patterns $1234,1243,1324$, and 2134 , where $r: \pi_{1} \pi_{2} \ldots \pi_{n} \rightarrow \pi_{n} \ldots \pi_{2} \pi_{1}$. So, for all $n \geq 0$,

$$
\# S_{n}(1234,1243,1324,2134)=\# S_{n}(4321,4231,4312,3421) .
$$

In this paper we give a complete answer for the number of freely-braided permutations in $\mathcal{F}_{n}$. The main result of this paper can be formulated as follows.

Theorem 1.3. The generating function for the number of freely-braided permutations in $\mathcal{F}_{n}$ is given by

$$
\frac{1-3 x-2 x^{2}+(1+x) \sqrt{1-4 x}}{1-4 x-x^{2}+\left(1-x^{2}\right) \sqrt{1-4 x}}=\frac{1}{1-4 x-x^{2}}\left(1-3 x-x^{2}+x^{3}-x^{2}(1+x)^{2} C(x)\right),
$$

where $C(x)=\frac{1-\sqrt{1-4 x}}{2 x}$ is the generating function for the Catalan numbers $\left(C_{n}=\frac{1}{n+1}\left(\begin{array}{c}2 n \\ n\end{array}\right)\right)$.

The proof of the above theorem is presented in Section 2

\section{Proof Theorem 1.3}

Given $b_{1}, b_{2}, \ldots, b_{m} \in \mathbb{N}$, we define

$$
f_{n}\left(b_{1}, b_{2}, \ldots, b_{m}\right)=\#\left\{\pi=\pi_{1} \pi_{2} \ldots \pi_{n} \in \mathcal{F}_{n} \mid \pi_{1} \pi_{2} \ldots \pi_{m}=b_{1} b_{2} \ldots b_{m}\right\} .
$$

It is natural to extend $f_{n}$ to the case $m=0$ by setting $f_{n}(\varnothing)=f_{n}=\# \mathcal{F}_{n}$. The following properties of the numbers $f_{n}\left(b_{1}, \ldots, b_{m}\right)$ can be deduced easily from the definitions.

\section{Lemma 2.1.}

(1) Let $m \geq 1$ and $n-2 \geq b_{1}>b_{2}>\cdots>b_{m} \geq 1$. Then, for all $b_{m}+1 \leq j \leq n-2$,

$$
f_{n}\left(b_{1}, \ldots, b_{m}, j\right)=0 .
$$

(2) Let $m \geq 2$ and $n-2 \geq b_{1}>b_{2}>\cdots>b_{m} \geq 1$. Then, for all $b_{m}+1 \leq j \leq n-1$,

$$
f_{n}\left(b_{1}, \ldots, b_{m}, j\right)=0 .
$$

(3) Let $m \geq 1$ and $n-2 \geq b_{1}>b_{2}>\cdots>b_{m} \geq 1$. Then

$$
f_{n}\left(b_{1}, \ldots, b_{m}, n\right)=f_{n-1}\left(b_{1}, \ldots, b_{m}\right) .
$$

(4) Let $m \geq 1$ and $n-2 \geq b_{1}>b_{2}>\cdots>b_{m} \geq 1$. Then

$$
f_{n}\left(n, b_{1}, \ldots, b_{m}\right)=f_{n}\left(n-1, b_{1}, \ldots, b_{m}\right)=f_{n-1}\left(b_{1}, \ldots, b_{m}\right) .
$$


(5) Let $m \geq 1$ and $n-1 \geq b_{1}>b_{2}>\cdots>b_{m} \geq 1$. Then

$$
f_{n}\left(b_{1}, n, \ldots, b_{m}\right)=f_{n-1}\left(b_{1}, \ldots, b_{m}\right) .
$$

Proof. For (1), observe that if $\pi \in S_{n}$ is such that $\pi_{1} \ldots \pi_{m} \pi_{m+1}=b_{1} \ldots b_{m} j$, then the entries $b_{m}, j, n-1$, $n$ give an occurrence of the pattern 1234 or 1243 in $\pi$.

For (2), observe that if $\pi \in S_{n}$ is such that $\pi_{1} \ldots \pi_{m} \pi_{m+1}=b_{1} \ldots b_{m} j$, then either the entries $b_{m-1}, b_{m}$, $n-1, n$ give an occurrence of the pattern 2134 in $\pi$ or the entries $b_{m}, j, n-1, n$ give an occurrence of 1234 or 1243 in $\pi$.

For (3), observe that if $\pi \in S_{n}$ is such that $\pi_{1} \ldots \pi_{m} \pi_{m+1}=b_{1} \ldots b_{m} n$, where $n-2 \geq b_{1}>\cdots>b_{m} \geq 1$, then no occurrence of the patterns 1234, 1243, 1324, 2134 in $\pi$ can involve the entry $\pi_{m+1}=n$. Hence, there is a bijection between the set of permutations $\pi \in \mathcal{F}_{n}$ with $\pi_{1} \ldots \pi_{m} \pi_{m+1}=b_{1} \ldots b_{m} n$ and the set of permutations $\sigma \in \mathcal{F}_{n-1}$ such that $\sigma_{1} \ldots \sigma_{m}=b_{1} \ldots b_{m}$.

Using similar arguments as in the proof of (3) we get that (4) and (5) hold.

Next we introduce objects $A_{m}(n), B_{m}(n)$ and $C_{m}(n)$ which organize suitably the information about the numbers $f_{n}\left(b_{1}, b_{2}, \ldots, b_{m}\right)$ and play an important role in the proof of the main result.

Definition 2.2. For $1 \leq m \leq n$ set

$$
\begin{aligned}
A_{m}(n) & =\sum_{n-2 \geq b_{1}>b_{2}>\cdots>b_{m} \geq 1} f_{n}\left(b_{1}, b_{2}, \ldots, b_{m}\right), \\
B_{m}(n) & =\sum_{n-2 \geq b_{1}>b_{2}>\cdots>b_{m} \geq 1} f_{n}\left(b_{1}, n-1, b_{2}, b_{3}, \ldots, b_{m}\right), \quad \text { and } \\
C_{m}(n) & =\sum_{n-1 \geq b_{1}>b_{2}>\cdots>b_{m} \geq 1} f_{n}\left(b_{1}, b_{2}, \ldots, b_{m}\right) .
\end{aligned}
$$

As before, this definition is extended to

the case $m=0$ by setting $A_{0}(n)=C_{0}(n)=f_{n}$ and $B_{0}(n)=0$.

In the following two subsections we derive expressions for $A_{m}(n)$ and $B_{1}(n)$, which are used in subsection 2.3 to complete the proof of Theorem 1.3

\subsection{A recurrence for the $f_{j}$, also involving $B_{1}(n)$}

In the following result we derive an expression for $A_{m}(n)$.

\section{Proposition 2.3.}

(1) For all $n \geq 2$,

$$
A_{1}(n)=f_{n}-2 f_{n-1} .
$$

(2) For all $n \geq 3$,

$$
A_{2}(n)=f_{n}-3 f_{n-1}+f_{n-2}-B_{1}(n) .
$$

(3) For all $2 \leq m \leq n-2$,

$$
A_{m}(n)=A_{m+1}(n)+A_{m}(n-1)+\cdots+A_{0}(n-1-m) .
$$


Proof. For (1), Definition 2.2 for $A_{1}(n)$ gives that

$$
A_{1}(n)=\sum_{b_{1}=1}^{n-2} f_{n}\left(b_{1}\right)=f_{n}-f_{n}(n-1)-f_{n}(n) .
$$

Observe that if $\pi \in S_{n}$ is such that $\pi_{1}=n-1$ or $\pi_{1}=n$, then no occurrence of the patterns 1234,1243 , 1324,2134 in $\pi$ can involve the entry $\pi_{1}$. So we get that the number of permutations in $\mathcal{F}_{n}$ starting with $n$ (resp., $n-1)$ is $f_{n-1}$. Hence, $A_{1}(n)=f_{n}-2 f_{n-1}$, as claimed in (1).

For 2), Definition 2.2 for $A_{1}(n)$ and $A_{2}(n)$ gives that

$$
A_{1}(n)=A_{2}(n)+\sum_{b_{1}=1}^{n-2} \sum_{b_{2}=b_{1}+1}^{n} f_{n}\left(b_{1}, b_{2}\right) .
$$

Using Lemma 2.1, parts (1) and (5), and Definition 2.2 we obtain that

$$
A_{1}(n)=A_{2}(n)+B_{1}(n)+A_{1}(n-1)+f_{n-1}(n-2) .
$$

Hence, by using the proof of (1) and Definition 2.2 we get the desired result.

For (3), let $2 \leq m \leq n-2$. Definition 2.2 yields

$$
A_{m}(n)=A_{m+1}(n)+\sum_{n-2 \geq b_{1}>b_{2}>\cdots>b_{m} \geq 1} \sum_{j=b_{m}+1}^{n} f_{n}\left(b_{1}, \ldots, b_{m}, j\right) .
$$

Using Lemma 2.1, parts (2) and (3), we have

$$
\begin{aligned}
A_{m}(n) & =A_{m+1}(n)+\sum_{n-2 \geq b_{1}>b_{2}>\cdots>b_{m} \geq 1} f_{n}\left(b_{1}, b_{2}, \ldots, b_{m}, n\right) \\
& =A_{m+1}(n)+\sum_{n-2 \geq b_{1}>b_{2}>\cdots>b_{m} \geq 1} f_{n-1}\left(b_{1}, b_{2}, \ldots, b_{m}\right) \\
& =A_{m+1}(n)+C_{m}(n-1) .
\end{aligned}
$$

Definition 2.2 and Lemma 2.1, (4) give

$$
\begin{aligned}
C_{m}(n) & =A_{m}(n)+\sum_{n-2 \geq b_{2}>\cdots>b_{m} \geq 1} f_{n}\left(n-1, b_{2}, \ldots, b_{m}\right) \\
& =A_{m}(n)+\sum_{n-2 \geq b_{2}>\cdots>b_{m} \geq 1} f_{n-1}\left(b_{2}, \ldots, b_{m}\right) \\
& =A_{m}(n)+C_{m-1}(n-1) .
\end{aligned}
$$

Hence, by induction on $m$ together with (1) we get the desired result.

We next find an explicit expression for $A_{m}(n)$ in terms of $A_{0}(n)=f_{n}, A_{1}(n)$ and $A_{2}(n)$.

Theorem 2.4. For all $n \geq 5$ and $2 \leq m \leq n-2$,

$$
A_{m}(n)=\sum_{j \geq 0}(-1)^{j}\left(\begin{array}{c}
m-1-j \\
j
\end{array}\right) A_{2}(n-j)-\sum_{j \geq 0}(-1)^{j}\left(\begin{array}{c}
m-3-j \\
j
\end{array}\right)\left(A_{1}(n-2-j)+A_{0}(n-3-j)\right),
$$

with the usual convention that $\left(\begin{array}{l}a \\ b\end{array}\right)=0$ if $a<b$ or $a<0$. 
Proof. For $m=2$ we have that $A_{2}(n)=A_{2}(n)$, so the theorem holds. Assume the theorem for $m$ and all appropriate $n$, and let us prove the equality for $m+1$. Using Proposition 2.3 .3 we get that

$$
A_{m+1}(n)=A_{m}(n)-\sum_{i=0}^{m} A_{m-i}(n-1-i)
$$

and by the induction hypothesis, we arrive at

$$
\begin{aligned}
& A_{m+1}(n) \\
& =\sum_{j \geq 0}(-1)^{j}\left(\begin{array}{c}
m-1-j \\
j
\end{array}\right) A_{2}(n-j)-\sum_{j \geq 0}(-1)^{j}\left(\begin{array}{c}
m-3-j \\
j
\end{array}\right)\left(A_{1}(n-2-j)+A_{0}(n-3-j)\right) \\
& \quad-\sum_{i=0}^{m} \sum_{j \geq 0}(-1)^{j}\left(\begin{array}{c}
m-1-i-j \\
j
\end{array}\right) A_{2}(n-1-i-j) \\
& \quad+\sum_{i=0}^{m} \sum_{j \geq 0}(-1)^{j}\left(\begin{array}{c}
m-3-i-j \\
j
\end{array}\right)\left(A_{1}(n-3-i-j)+A_{0}(n-4-i-j)\right) \\
& =\sum_{j \geq 0}(-1)^{j}\left(\begin{array}{c}
m-1-j \\
j
\end{array}\right) A_{2}(n-j)-\sum_{j \geq 0}(-1)^{j}\left(\begin{array}{c}
m-3-j \\
j
\end{array}\right)\left(A_{1}(n-2-j)+A_{0}(n-3-j)\right) \\
& \quad-\sum_{j \geq 0}\left(\begin{array}{c}
j \\
i=0
\end{array}(-1)^{i}\left(\begin{array}{c}
m-1-j \\
i
\end{array}\right) A_{2}(n-1-j)-\sum_{i=0}^{j}(-1)^{i}\left(\begin{array}{c}
m-3-j \\
i
\end{array}\right)\left(A_{1}(n-3-j)+A_{0}(n-4-j)\right)\right) .
\end{aligned}
$$

Using the familiar identity $\left(\begin{array}{l}p \\ 0\end{array}\right)-\left(\begin{array}{c}p \\ 1\end{array}\right)+\cdots+(-1)^{q}\left(\begin{array}{l}p \\ q\end{array}\right)=(-1)^{q}\left(\begin{array}{c}p-1 \\ q\end{array}\right)$ we obtain that

$$
\begin{aligned}
A_{m+1}(n) & =\sum_{j \geq 0}(-1)^{j}\left(\begin{array}{c}
m-1-j \\
j
\end{array}\right) A_{2}(n-j)-\sum_{j \geq 0}(-1)^{j}\left(\begin{array}{c}
m-3-j \\
j
\end{array}\right)\left(A_{1}(n-2-j)+A_{0}(n-3-j)\right) \\
& -\sum_{j \geq 0}(-1)^{j}\left(\begin{array}{c}
m-2-j \\
j
\end{array}\right) A_{2}(n-1-j)+\sum_{j \geq 0}(-1)^{j}\left(\begin{array}{c}
m-4-j \\
j
\end{array}\right)\left(A_{1}(n-3-j)+A_{0}(n-4-j)\right),
\end{aligned}
$$

and by using the identity $\left(\begin{array}{c}p \\ q\end{array}\right)=\left(\begin{array}{c}p-1 \\ q\end{array}\right)+\left(\begin{array}{c}p-1 \\ q-1\end{array}\right)$ we get that

$$
A_{m+1}(n)=\sum_{j \geq 0}(-1)^{j}\left(\begin{array}{c}
m-j \\
j
\end{array}\right) A_{2}(n-j)-\sum_{j \geq 0}(-1)^{j}\left(\begin{array}{c}
m-2-j \\
j
\end{array}\right)\left(A_{1}(n-2-j)+A_{0}(n-3-j)\right) .
$$

Hence, by induction on $m$ we get the desired result.

Using Theorem 2.4 for $m=n-2$, together with Proposition 2.3. parts (1) and 2), and $A_{n-2}(n)=1$ (see Definition 2.2 we get the main result of this subsection.

Theorem 2.5. For all $n \geq 5$,

$\sum_{j \geq 0}(-1)^{j}\left(\begin{array}{c}n-3-j \\ j\end{array}\right)\left(f_{n-j}-3 f_{n-1-j}+f_{n-2-j}-B_{1}(n-j)\right)=1+\sum_{j \geq 0}(-1)^{j}\left(\begin{array}{c}n-5-j \\ j\end{array}\right)\left(f_{n-2-j}-f_{n-3-j}\right)$.

\subsection{A recursive formula for $B_{1}(n)$}

We next we find a recurrence for $B_{1}(n)$ in terms of $f_{n}$.

Proposition 2.6. We have

$B_{1}(n)=C_{0}(n-2)+C_{1}(n-2)+\cdots+C_{n-2}(n-2)$ for all $n \geq 3$. 
$B_{1}(n)-B_{1}(n-1)=A_{0}(n-2)+A_{1}(n-2)+\cdots+A_{n-4}(n-2)$ for all $n \geq 4$.

Proof. For 2.6), by Definition 2.2 we get that

$$
B_{1}(n)=f_{n}(n-2, n-1)+\sum_{n-3 \geq b_{1} \geq 1} f_{n}\left(b_{1}, n-1\right) .
$$

Observe that if a permutation $\pi \in S_{n}$ is such that $\pi_{1}=n-2$ and $\pi_{2}=n-1$, then no occurrence of the patterns $1234,1243,1324,2134$ can involve either the entry $n-1$ or the entry $n$. Thus, $f_{n}(n-2, n-1)=$ $f_{n-2}=C_{0}(n-2)$, and for all $n \geq 3, B_{1}(n)=C_{0}(n-2)+L_{1}(n)$, where we define

$$
L_{m}(n)=\sum_{n-3 \geq b_{1}>\cdots>b_{m} \geq 1} f_{n}\left(b_{1}, n-1, b_{2}, \ldots, b_{m}\right) \quad \text { for } m \geq 1 .
$$

Using Lemma 2.1, parts (1) and (2), we get that

$$
L_{m}(n)=L_{m+1}(n)+\sum_{n-3 \geq b_{1}>\cdots>b_{m} \geq 1} f_{n}\left(b_{1}, n-1, b_{2}, \ldots, b_{m}, n\right),
$$

and by Lemma 2.1, parts (3) and (5), together with Definition 2.2 we arrive at

$$
L_{m}(n)=L_{m+1}(n)+C_{m}(n-2)
$$

for all $m \geq 1$. Hence, by induction on $m$ together with the fact that $L_{n-1}(n)=0$ we have

$$
B_{1}(n)=C_{0}(n-2)+C_{1}(n-2)+\cdots+C_{n-2}(n-2),
$$

as claimed.

For (2.6), using Equation (2.1) together with 2.6) and $C_{n}(n)=A_{n-1}(n)=0$ (see Definition 2.2 we get the desired result.

Theorem 2.7. For all $n \geq 4$,

$$
\begin{aligned}
& B_{1}(n)-B_{1}(n-2)=f_{n-1}-2 f_{n-2}+f_{n-3} \\
& \quad-\sum_{j \geq 0}(-1)^{j}\left(\begin{array}{c}
n-3-j \\
j
\end{array}\right) A_{2}(n-1-j)+\sum_{j \geq 0}(-1)^{j}\left(\begin{array}{c}
n-5-j \\
j
\end{array}\right)\left(A_{1}(n-3-j)+A_{0}(n-4-j)\right) .
\end{aligned}
$$

Proof. It is easy to check that the theorem holds for $n=4,5,6$. Now, let $n \geq 7$. By using Proposition 2.6 2.6 and Theorem 2.4 we get that

$$
\begin{aligned}
B_{1}(n)-B_{1}(n-1)= & A_{0}(n-2)+A_{1}(n-2)+\sum_{m=2}^{n-4} \sum_{j \geq 0}(-1)^{j}\left(\begin{array}{c}
m-1-j \\
j
\end{array}\right) A_{2}(n-2-j) \\
& -\sum_{m=3}^{n-4} \sum_{j \geq 0}(-1)^{j}\left(\begin{array}{c}
m-3-j \\
j
\end{array}\right)\left(A_{1}(n-4-j)+A_{0}(n-5-j)\right) \\
= & A_{0}(n-2)+A_{1}(n-2)-A_{2}(n-2)+\sum_{m=1}^{n-4} \sum_{j \geq 0}(-1)^{j}\left(\begin{array}{c}
m-1-j \\
j
\end{array}\right) A_{2}(n-2-j) \\
& -\sum_{m=3}^{n-4} \sum_{j \geq 0}(-1)^{j}\left(\begin{array}{c}
m-3-j \\
j
\end{array}\right)\left(A_{1}(n-4-j)+A_{0}(n-5-j)\right) \\
= & A_{0}(n-2)+A_{1}(n-2)-A_{2}(n-2)+\sum_{j \geq 0} \sum_{i=j}^{n-5-j}(-1)^{j}\left(\begin{array}{l}
i \\
j
\end{array}\right) A_{2}(n-2-j) \\
& -\sum_{j \geq 0} \sum_{i=j}^{n-7-j}(-1)^{j}\left(\begin{array}{c}
i \\
j
\end{array}\right)\left(A_{1}(n-4-j)+A_{0}(n-5-j)\right) .
\end{aligned}
$$


Therefore, using the identity $\left(\begin{array}{c}p \\ p\end{array}\right)+\left(\begin{array}{c}p+1 \\ p\end{array}\right)+\cdots+\left(\begin{array}{c}q \\ p\end{array}\right)=\left(\begin{array}{c}q+1 \\ p+1\end{array}\right)$ gives that

$$
\begin{aligned}
& B_{1}(n)-B_{1}(n-1) \\
& \quad=A_{0}(n-2)+A_{1}(n-2)-A_{2}(n-2)+A_{2}(n-1)-A_{1}(n-3)-A_{0}(n-4) \\
& \quad-\sum_{j \geq 0}(-1)^{j}\left(\begin{array}{c}
n-3-j \\
j
\end{array}\right) A_{2}(n-1-j)+\sum_{j \geq 0}(-1)^{j}\left(\begin{array}{c}
n-5-j \\
j
\end{array}\right)\left(A_{1}(n-3-j)+A_{0}(n-4-j)\right) .
\end{aligned}
$$

Hence, using Proposition 2.3. parts (1) and (2), we obtain the desired identity.

\subsection{Proof of Theorem 1.3}

We start by showing the following result.

Lemma 2.8. Let $t(x)$ be the generating function for the sequence $\left(t_{n}\right)_{n \geq 0}$, that is, $t(x)=\sum_{n \geq 0} t_{n} x^{n}$. Then

$$
\sum_{n \geq m}\left(x^{n} \sum_{j \geq 0}(-1)^{j}\left(\begin{array}{c}
n-m-j \\
j
\end{array}\right) t_{n-s-j}\right)=\frac{x^{s}}{(1-x)^{m-s}}\left(t(x(1-x))-\sum_{j=0}^{m-s-1} t_{j} x^{j}(1-x)^{j}\right) .
$$

Proof. We have

$$
\begin{aligned}
\sum_{n \geq m}\left(x^{n} \sum_{j \geq 0}(-1)^{j}\left(\begin{array}{c}
n-m-j \\
j
\end{array}\right) t_{n-s-j}\right) & =\sum_{n \geq 0} \sum_{j=0}^{n}(-1)^{j}\left(\begin{array}{l}
n \\
j
\end{array}\right) x^{n+m+j} t_{n+m-s} \\
=\sum_{n \geq 0} t_{n+m-s} x^{n+m}(1-x)^{n} & =\frac{x^{s}}{(1-x)^{m-s}}\left(t(x(1-x))-\sum_{j=0}^{m-s-1} t_{j} x^{j}(1-x)^{j}\right),
\end{aligned}
$$

as claimed.

Now we are ready to prove the main result of this paper, namely Theorem 1.3 , which is restated here for easy reference.

Theorem 1.3 The generating function for the number of freely-braided permutations in $\mathcal{F}_{n}$ is given by

$$
\frac{1-3 x-2 x^{2}+(1+x) \sqrt{1-4 x}}{1-4 x-x^{2}+\left(1-x^{2}\right) \sqrt{1-4 x}}
$$

Proof. We denote the generating function for the number of freely-braided permutations in $\mathcal{F}_{n}$ by $F(x)$, that is, $F(x)=\sum_{n \geq 0} f_{n} x^{n}$. Also, we denote the generating function for the sequence $\left\{B_{1}(n)\right\}_{n \geq 0}$ by $B(x)$, that is, $B(x)=\sum_{n \geq 0} B_{1}(n) x^{n}$.

Theorem 2.5 gives

$$
\sum_{j \geq 0}(-1)^{j}\left(\begin{array}{c}
n-3-j \\
j
\end{array}\right)\left(f_{n-j}-3 f_{n-1-j}+f_{n-2-j}-B_{1}(n-j)\right)=1+\sum_{j \geq 0}(-1)^{j}\left(\begin{array}{c}
n-5-j \\
j
\end{array}\right)\left(f_{n-2-j}-f_{n-3-j}\right),
$$

for all $n \geq 5$. Multiplying by $x^{n}$ and summing over all $n \geq 5$ together with using Lemma 2.8 we arrive at 


$$
\begin{aligned}
& -x^{4}+\frac{1}{(1-x)^{3}}\left(\left(1-3 x(1-x)+x^{2}(1-x)^{2}\right) F(x(1-x))-1+2 x(1-x)-B(x(1-x))\right) \\
& \quad=\frac{x^{5}}{1-x}+\frac{x^{2}}{(1-x)^{3}}\left(F(x(1-x))-1-x(1-x)-2 x^{2}(1-x)^{2}\right)+\frac{x^{2}}{(1-x)^{2}}(F(x(1-x))-1-x(1-x)),
\end{aligned}
$$

or equivalently,

$$
F(x(1-x))-\frac{1}{(1-x)^{3}} B(x(1-x))=\frac{1}{1-x} .
$$

Theorem 2.7 gives

$$
\begin{aligned}
& B_{1}(n)-B_{1}(n-2)=f_{n-1}-2 f_{n-2}+f_{n-3} \\
& \quad-\sum_{j \geq 0}(-1)^{j}\left(\begin{array}{c}
n-3-j \\
j
\end{array}\right) A_{2}(n-1-j)+\sum_{j \geq 0}(-1)^{j}\left(\begin{array}{c}
n-5-j \\
j
\end{array}\right)\left(A_{1}(n-3-j)+A_{0}(n-4-j)\right),
\end{aligned}
$$

for all $n \geq 4$. Multiplying by $x^{n}$ and summing over all $n \geq 4$ together with using Lemma 2.8 we arrive at

$$
\begin{aligned}
& \left(1-x^{2}\right) B(x)-x^{3}=\frac{x}{(1-x)^{2}} F(x)-x+x^{2}(1-x) \\
& \quad-\frac{x}{(1-x)^{2}}\left(\left(1-3 x(1-x)+x^{2}(1-x)^{2}\right) F(x(1-x))-1+2 x(1-x)-B(x(1-x))\right) \\
& \quad+\frac{x^{3}}{(1-x)^{2}}(F(x(1-x))-1-x(1-x))-\frac{x^{4}}{1-x}(F(x(1-x)-1),
\end{aligned}
$$

or equivalently,

$$
\left(1-x^{2}\right) B(x)=x^{2}-x(1-x) F(x(1-x))+x(1-x)^{2} F(x)+\frac{x}{(1-x)^{2}} B(x(1-x)) .
$$

Using Equations 2.2 and 2.3 we get that

$$
\left\{\begin{array}{l}
B(x(1-x))=(1-x)^{3} F(x(1-x))-(1-x)^{2} \\
(1+x) B(x)=-x+x(1-x) F(x)
\end{array}\right.
$$

or equivalently,

$$
\left\{\begin{array}{ll}
B(x) & =\left(1-\frac{1-\sqrt{1-4 x}}{2}\right)^{3} F(x)-\left(1-\frac{1-\sqrt{1-4 x}}{2}\right)^{2} \\
(1+x) B(x) & =-x+x(1-x) F(x)
\end{array} .\right.
$$

The rest is easy to check.

\section{Acknowledgements}

I would like to thank R.M. Green and J. Losonczy for bringing to my attention the problem of finding an explicit formula for $\# S_{n}(4321,3421,4231,4312)$. Special thanks to J. Losonczy for his helpful comments. 


\section{References}

[1] M. D. Atkinson, Permutations which are the union of an increasing and decreasing subsequence, Electron. J. Combin 5 (1998), Article \#R6.

[2] E. Barcucci, A. D. Lungo, E. Pergola, and R. Pinzani, Permutations avoiding and increasing number of length-increasing forbidden subsequences, Discrete Math. Theor. Comput. Sci. 4 (2000), 31-44.

[3] M. Bóna, Exact enumeration of 1342-avoiding permutations: A close link with labeled trees and planar maps, J. Combin. Theory, Series A 80 (1997), 257-272.

[4] M. Bóna, The permutation classes equinumerous to the smooth class, Electron. J. Combin. 5 (1998), Article \#R31.

[5] R.M. Green and J. Losonczy, Freely braided elements in Coxeter groups Annals of Combinatorics 6 (2002), 337-348.

[6] R.M. Green and J. Losonczy, Freely braided elements in Coxeter groups II, Advances in Applied Mathematics 33 (2004), 26-39.

[7] O. Guibert, "Permutations sans sous-séquence interdite”, PhD thesis, Université Bordeaux I, 1992.

[8] D. Kremer, Permutations with forbidden subsequences and a generalized Schröder number, Discrete Math. 218 (2000), 121-130.

[9] D. Kremer and W. C. Shiu, Finite transition matrices for permutations avoiding pairs of length four patterns. Discrete Math. 268 (2003), 171-183.

[10] T. Mansour, Permutations avoiding a pattern from $S_{k}$ and at least two patterns from $S_{3}$, Ars Combin., 62 (2001), 227-239..

[11] T. Mansour and A. Vainshtein, Layered restrictions and Chebyshev polynomials, Annals of Combinatorics 5 (2001), 451-458.

[12] T. Mansour and A. Vainshtein, Avoiding maximal parabolic subgroups of $S_{k}$, Discrete Math. Theor. Comput. Sci. 4 (2000), 67-75.

[13] T. Mansour and A. Vainshtein, Restricted 132-avoiding permutations, Adv. in Appl. Math. 26 (2001), 258-269.

[14] T. Mansour and A. Vainshtein, Restricted permutations and Chebyshev polynomials, Séminaire Lotharingien de Combinatoire 47 (2002), Article B47c.

[15] R. Simion, F.W. Schmidt, Restricted Permutations, Europ. J. of Combinatorics 6 (1985), 383-406.

[16] Z. E. Stankova, Forbidden subsequences, Discrete Math. 132 (1994), 291-316.

[17] Z. E. Stankova, Classification of forbidden subsequences of length 4, Europ. J. Combin. 17 (1996), 501-517. 
[18] Z. Stankova-Frenkel and J. West, Explicit enumeration of 321-hexagon-avoiding permutations, Discr. Math. 280 (2004), 165-189.

[19] J. West, Generating trees and the Catalan and Schröder numbers, Discrete Math. 146 (1995), 247262.

[20] J. West, Generating trees and forbidden subsequences Discrete Math. 157 (1996), 363-374. 